\title{
Prospective phase II trial of preresection thoracoscopic mediastinal restaging after neoadjuvant therapy for IIIA (N2) non-small cell lung cancer: Results of CALGB Protocol 39803
}

\author{
Michael T. Jaklitsch, MD, ${ }^{\mathrm{a}}$ Lin Gu, MS, ${ }^{\mathrm{b}}$ Todd Demmy, MD, ${ }^{\mathrm{c}}$ David H. Harpole, MD, ${ }^{\mathrm{d}}$ \\ Thomas A. D’Amico, MD, ${ }^{\mathrm{d}}$ Robert J. McKenna, MD, ${ }^{\mathrm{e}}$ Mark J. Krasna, MD, ${ }^{\mathrm{f}}$ Leslie J. Kohman, MD, ${ }^{\mathrm{g}}$

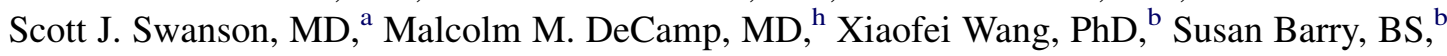 \\ David J. Sugarbaker, MD, ${ }^{\mathrm{a}}$ and the CALGB Thoracic Surgeons
}

Objective: Accurate pathologic restaging of N2 stations after neoadjuvant therapy in stage IIIA (N2) non-small cell lung cancer is needed.

Methods: A prospective multi-institutional trial was designed to judge the feasibility of videothoracoscopy to
restage the ipsilateral nodes in mediastinoscopy-proven stage IIIA (N2) non-small cell lung cancer after 2 cycles
of platinum-based chemotherapy and/or 40 Gy or more of radiotherapy. The goals included biopsy of 3 negative
N2 node stations or to identify 1 positive N2 node or pleural carcinomatosis.

Results: Ten institutions accrued 68 subjects. Of the 68 subjects, $46(68 \%)$ underwent radiotherapy and 66 $(97 \%)$ underwent chemotherapy. Videothoracoscopy successfully met the prestudy feasibility in 27 patients (40\%): 3 negative stations confirmed at thoracotomy in 7, persistent stage N2 disease in 16, and pleural carcinomatosis in 4 . In 20 procedures $(29 \%)$, no N2 disease was found, 3 stations were not biopsied because of unanticipated nodal obliteration. Thus, 47 videothoracoscopy procedures $(69 \%, 95 \%$ confidence interval, $57 \%-80 \%)$ restaged the mediastinum. Videothoracoscopy was unsuccessful in 21 patients $(31 \%)$ because the procedure had to be aborted $(n=11)$ or because of false-negative stations $(n=10)$. Of the 21 failures, 15 were right-sided, and 10 had a positive $4 \mathrm{R}$ node. The sensitivity of videothoracoscopy was $67 \%(95 \%$ confidence interval, $47 \%-83 \%)$, and the negative predictive value was $73 \%(95 \%$ confidence interval, $56 \%-86 \%)$ if patients with obliterated nodal tissue were included. The sensitivity was $83 \%(95 \%$ confidence interval, $63 \%$ $95 \%)$ and the negative predictive value was $64 \%$ (95\% confidence interval, $31 \%-89 \%)$ if those patients were excluded. The specificity was $100 \%$. One death occurred after thoracotomy.

Conclusions: Videothoracoscopy restaging was "feasible" in this prospective multi-institutional trial and provided pathologic specimens of the ipsilateral nodes. Videothoracoscopy restaging was limited by radiation and the 4R nodal station. (J Thorac Cardiovasc Surg 2013;146:9-16)

The Cancer and Leukemia Group B (CALGB) 8935 study helped to establish the role of standardized pretreatment histopathologic staging by mediastinoscopy before neoadjuvant

\footnotetext{
From the Brigham and Women's Hospital, ${ }^{\mathrm{a}}$ Boston, Mass; CALGB Statistical Center, ${ }^{\mathrm{b}}$ Durham, NC; Roswell Park Cancer Institute, ${ }^{\mathrm{c}}$ Buffalo, NY; Duke University, ${ }^{\mathrm{d}}$ Durham, NC; Cedars-Sinai Hospital, ${ }^{\mathrm{e}}$ Los Angeles, Calif; Meridian Cancer Center, ${ }^{\mathrm{f}}$ Neptune, NJ; Upstate Medical University, Syracuse, ${ }^{\mathrm{g}} \mathrm{NY}$; and Northwestern University, ${ }^{\text {h }}$ Chicago, Ill.

The research for CALGB 39803 was supported, in part, by grants from the National Cancer Institute (CA31946) to the Cancer and Leukemia Group B (Monica M. Bertagnolli, MD, Chair) and to the CALGB Statistical Center (Daniel J. Sargent, PhD, CA33601).

The content of this report is solely the responsibility of the authors and does not necessarily represent the official views of the National Cancer Institute.

Disclosures: Authors have nothing to disclose with regard to commercial support.

A complete list of the institutions participating in this study can be found in the Appendix.

Received for publication July 23, 2012; revisions received Dec 7, 2012; accepted for publication Dec 18, 2012

Address for reprints: Michael T. Jaklitsch, MD, Division of Thoracic Surgery, Brigham and Women's Hospital, 75 Francis St, Boston, MA 02115 (E-mail: mjaklitsch@partners.org).

$0022-5223 / \$ 36.00$

Copyright (C) 2013 by The American Association for Thoracic Surgery

http://dx.doi.org/10.1016/j.jtcvs.2012.12.069
}

therapy for stage IIIA (N2) non-small cell lung cancer (NSCLC). ${ }^{1}$ Mature follow-up data from both the CALGB 8935 study and the Southwest Oncology Group 8805, 2 independent, prospective, multi-institutional studies, indicated that patients with residual $\mathrm{N} 2$ disease at surgical resection after induction therapy did not enjoy the same survival as those with effective nodal downstaging. ${ }^{2,3}$ Specifically, in the Southwest Oncology Group 8805 study, a significant difference was seen in median survival between those with no $\mathrm{N} 2$ nodal disease at thoracotomy (30 months) and those with persistent N2 disease (10 months, $P=.005) .{ }^{2}$ Likewise, in the CALGB 8935 study, the median failure-free survival after a postsurgical 3-month landmark (to account for heterogenous adjuvant treatments) was 47.8 months if downstaged to no disease in the $\mathrm{N} 2$ nodes at thoracotomy compared with 8.2 months if persistent disease was present $(P=.01){ }^{3}$ Such patients rarely benefited from surgical resection, which sometimes caused perioperative morbidity, mortality, and temporary loss of independence.

Accordingly, we need a safe, accurate, minimally invasive method to restage the mediastinal nodes after neoadjuvant 


\section{Abbreviations and Acronyms \\ CALGB $=$ Cancer and Leukemia Group B \\ EBUS = endobronchial ultrasound \\ EUS = endoesophageal ultrasound \\ NPV = negative predictive value \\ NSCLC $=$ non-small cell lung cancer \\ PET = positron emission tomography \\ VATS $=$ videothoracoscopy}

therapy to allow the separation of patients expected to benefit from surgical resection from those with persistent nodal disease. Pathologic restaging of the mediastinal nodes could prevent futile thoracotomy for those not responding to induction therapy. Repeat mediastinoscopy has been associated with an increased risk of complications and reduced accuracy because the paratracheal spaces, subcarinal space, and aortopulmonary window are fused after previous dissection, radiotherapy, or cytotoxic therapy. ${ }^{4,5}$

Thoracoscopy is an attractive alternative to repeat mediastinoscopy because of its enhanced visibility and access to the posterior mediastinal, inferior mediastinal, retrohilar, and aortopulmonary window nodes. Although thoracoscopy can also discover clinically occult pleural carcinomatosis, it limits the surgeon to an ipsilateral assessment of the mediastinum.

Our hypothesis was that thoracoscopy could be used to provide pathologic restaging of the ispislateral nodes after neoadjuvnat therapy in those patients proved to have pathologic involvement of those nodes before treatment. Our primary endpoint was to determine the safety and feasibility of accessing multiple nodal stations thoracoscopically and to validate apparent downstaging with thoracotomy. If the technique of thoracoscopic restaging can be validated in the present feasibility study, the concept could become an important piece of the future strategy of CALGB activity, as multimodality therapy for stage III NSCLC is refined.

\section{METHODS}

A prospective, nonrandomized phase II trial was designed to evaluate the feasibility of videothoracoscopy (VATS) to assess resectability in patients with stage IIIA (N2) NSCLC after previous mediastinoscopy and neoadjuvant therapy by the CALGB group, a National Cancer Institutesponsored multi-institutional cooperative group. Unresectability was defined as the presence of a persistently positive mediastinal (N2) lymph node or pleural carcinomatosis. Secondary objectives included documentation of safety and assessment of the accuracy (false-negative rate) of preresection thoracoscopic mediastinal node restaging after previous mediastinoscopy and induction therapy.

Eligibility required histologic documentation of stage IIIA (N2) by mediastinoscopy performed before induction therapy. At least 2 cycles of chemotherapy with or without radiotherapy ( $\geq 40$ Gy) or radiotherapy alone ( $\geq 40 \mathrm{~Gy}$ ) had to be completed within 60 days of registration. Patients with responding or stable local disease were eligible.

Patients were ineligible for the present trial if they had a comorbid illness that made them unsuitable for subsequent resection, if they were unwilling to undergo subsequent anatomic resection (ie, lobectomy or pneumonectomy), had postinduction spirometry, which suggested they could not undergo surgery, or had a currently active second malignancy other than a nonmelanoma skin cancer. Patients with previously treated relapsed lung cancer were not eligible. Those with local disease progression, defined as a $25 \%$ increase in local tumor size or the appearance of new areas of malignant disease, were also ineligible. The patients could not have a history of intrapleural surgery on the ipsilateral side. An Eastern Cooperative Oncology Group performance status of 0 to 2 was required.

All patients provided informed consent, and the individual institutional review board at each participating institution approved the study. Before registration, the patients had to be evaluated by a CALGB credentialed thoracic surgeon and be determined suitable for surgical resection of their cancer. The surgeons were considered credentialed if they had accrued patients to previous CALGB VATS protocols. New surgeons were credentialed by the principal investigator after submitting the operative notes of 10 minimally invasive procedures that reflected a skill level necessary to participate in the present protocol.

Before making the thoracotomy incision to resect the lung after neoadjuvant therapy, patients underwent ipsilateral VATS. The patient was positioned in a lateral decubitus position after selective lung ventilation was established. Three to four thoracoscopic ports were recommended, although the placement of ports was left to surgeon discretion. In general, the camera port was placed in the 6th or 7th intercostal space, and the pleural space was evaluated for adhesions. Additional operative ports were recommended to be in line with the planned thoracotomy incision, including 1 anterior to the scapular tip and 1 at the posterior extent. A fourth port, if needed, was generally placed in the axilla.

Thoracoscopic restaging was considered positive if histologic proof was present of pleural carcinomatosis, malignant effusion, or any positive mediastinal node. The diseased nodal station identified by pretreatment mediastinoscopy was a specific target for restaging, unless pleural carcinomatosis or another positive mediastinal nodal station was identified before sampling that particular station. The procedure was considered complete and negative if no evidence was found of unresectability, at least 3 nodal stations were sampled, and no evidence of malignant disease was found.

The protocol guided the surgeon to restage specific nodal stations as a function of the primary tumor location. Tumors in the right upper lobe were to have stations $2 \mathrm{R}, 4 \mathrm{R}$, and 7 sampled. Tumors of the right middle and right lower lobes were to have biopsy samples taken of stations $4 R$, 7 , and $9 R$, and samples of station $2 R$ were encouraged but optional. Right hilar tumors were to have samples from stations $2 \mathrm{R}, 4 \mathrm{R}, 7$, and $9 \mathrm{R}$. Left upper lobe tumors were to have biopsy samples of stations 5,6 , and 7 and samples of station $4 \mathrm{~L}$ were encouraged "if feasible" (wording of the protocol). Left lower lobe tumors were to be restaged by sampling stations $5,7,9 \mathrm{~L}$, and biopsy samples of station $4 \mathrm{~L}$ were optional. Likewise, left hilar tumors were to have biopsy samples of stations $5,6,7$, or $9 \mathrm{~L}$, with optional sampling of station 4. At thoracotomy, these same nodal stations were to be sampled. In particular, the original positive nodal station identified at pretreatment cervical mediastinoscopy was a specific target at thoracotomy.

Most patients with negative restaging findings underwent thoracotomy and anatomic resection (ie, lobectomy, bilobectomy, or pneumonectomy) with mediastinal lymphadenectomy under the same anesthetic. Seven patients underwent VATS restaging and thoracotomy as 2 separate procedures. The interval ranged from 6 to 29 days. Patients with positive restaging findings could still undergo resection at the discretion of the surgeon.

\section{Statistical Considerations}

Patient registration and data collection were managed by the CALGB Statistical Center. Data quality was ensured by CALGB Statistical Center staff and by the study chair. Statistical analyses were performed by CALGB statisticians using SAS, version 9.1 (SAS Institute Inc, Cary, NC). 
Feasibility was defined as the ability to sample at least 3 negative mediastinal (N2) lymph node stations or to identify 1 positive mediastinal lymph node or pleural carcinomatosis. This feasibility protocol ended at the completion of surgery with either the finding of persistent malignancy or successful surgical resection. Long-term survival and recurrence patterns were not endpoints of the present study.

At the outset of the study, it was agreed that additional development of the surgical technique would be of interest if greater than $80 \%$ of patients had a successful procedure; additional investigation of the technique would not be warranted if fewer than $65 \%$ had a successful procedure unless significant modifications were done. With a planned accrual of 75 patients, there would be $90 \%$ power to differentiate a true feasibility rate of $65 \%$ versus $80 \%$ at a 1 -sided significance level of .05 .

The feasibility rate of VATS restaging and its $95 \%$ confidence interval (CI) are provided. The sensitivity and specificity of VATS restaging were calculated with the outcome of thoracotomy as the reference standard. The negative predictive value (NPV) and positive predictive value of VATS restaging were also calculated. All reported $P$ values are 2 sided. Grade 3 and greater complications were tabulated. The potential variables influencing VATS restaging success were evaluated using logistic regression analysis.

\section{RESULTS}

From activation on September 15, 1998, to closure on September 30, 2003, the accrual target was met by 75 participants from 10 institutions. Seven patients were unevaluable. Three subjects had undergone positron emission tomography (PET) staging of IIIA (N2) disease only, without pretreatment mediastinoscopy. The other 4 reasons (1 each) were a change in the postresection pathologic identification of hemangioendothelioma instead of lung cancer, a positive mediastinoscopy node that proved to be contralateral N3 disease and not an ipsilateral N2 mediastinal node (thus providing no pretreatment target to restage), PET staging without mediastinoscopy before neoadjuvant treatment (thus no pathologically confirmed target for restaging), and bulky hilar disease with direct invasion into the mediastinum that precluded subsequent surgical resection. The subsequent analysis was based on the data from 68 patients (Figure 1).

The demographic data are summarized in Table 1. Most participants were white, and $49 \%$ were men. The median age of this cohort was 61 years (range, 40-77 years). The Eastern Cooperative Oncology Group performance status was 0 in $60 \%$ and 1 in $40 \%$. All patients had undergone either neoadjuvant chemotherapy $(97 \%$ received $\geq 1$ cycle of chemotherapy, and $49 \%$ received 3 cycles) or radiotherapy $(68 \%)$, or both, before enrollment. About one half $(49 \%)$ of the participants were diagnosed with adenocarcinoma. Of the 68 patients, $53(78 \%)$ had just 1 positive mediastinal nodal station on initial mediastinoscopy, 14 (21\%) had 2 positive $\mathrm{N} 2$ nodal stations, and $1(1 \%)$ had 3 positive $\mathrm{N} 2$ nodal stations. Thus, 15 of the $68(22 \%)$ evaluable participants had multistation N2 disease; a population that many would not consider for resection outside of an investigational protocol.

Thoracoscopic restaging was performed on the right side in $75 \%$ of the participants. The median number of thoracoscopic incisions was 3 (range, 1-4). The median duration of the VATS restaging procedure was 63 minutes (range, 15-313 minutes). Thoracotomy was performed in 61 patients. The 4 patients found to have pleural carcinomatosis and 3 of those found to have persistent nodal disease did not undergo subsequent thoracotomy.

Postoperative morbidity data were available for $66 \mathrm{pa}-$ tients and are summarized in Table 2. One operative death (only case of grade 5 toxicity) occurred in the present study of acute respiratory distress syndrome after subsequent thoracotomy for lung resection. A total of 9 individual grade 3 toxicities were noted in 7 patients after a combination of thoracoscopic restaging followed by thoracotomy and attempted anatomic resection. One patient experienced a biopsy forceps injury to the left main bronchus (grade 3 toxicity) during restaging of the station 7 subcarinal nodes. The restaging procedure was abandoned in this patient, and thoracotomy was performed to repair the recognized injury. A grade 4 cardiovascular arrhythmia and a grade 4 thrombosis occurred in the same patient who died of acute respiratory distress syndrome (grade 5).

The median interval with a chest tube in place was 4 days (range, 1-72 days). Prolonged chest tube duration was attributed to air leaks from the anatomic resection in $88 \%$ of evaluable patients with stage IIIA disease (all of whom had undergone neoadjuvant therapy) rather than from the restaging procedure.

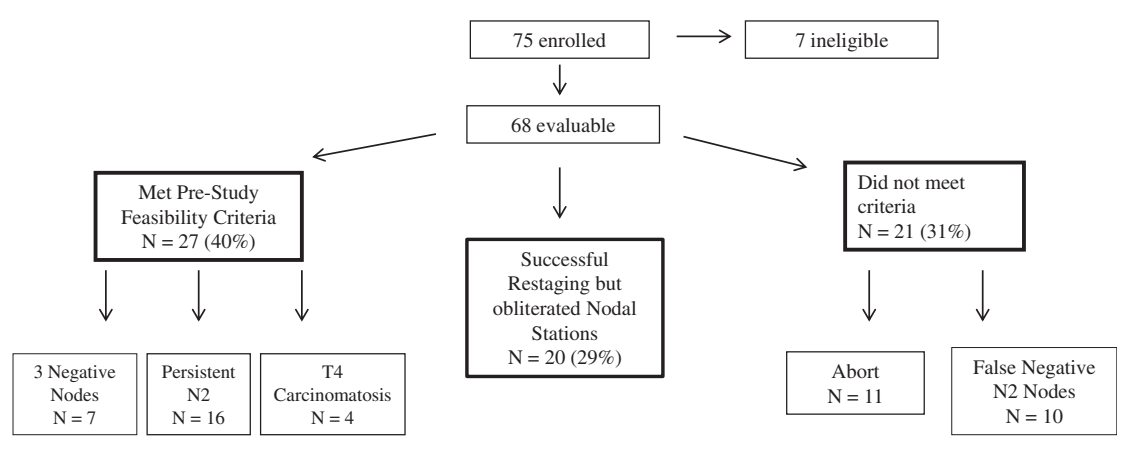

FIGURE 1. Flow sheet of patient outcomes. 
TABLE 1. Patient demographic and baseline clinical characteristics $(\mathbf{n}=68)$

\begin{tabular}{lc}
\hline \multicolumn{1}{c}{ Variable } & Value \\
\hline Gender & \\
Male & $33(49)$ \\
Female & $35(51)$ \\
Age (y) & \\
$\quad$ Median & 61 \\
Range & $40-77$ \\
Race & \\
White & $66(97)$ \\
Black & $1(1)$ \\
Asian & $1(1)$ \\
Performance status & \\
0 & $41(60)$ \\
1 & $27(40)$ \\
Previous treatment & \\
Thoracic radiotherapy & $46(68)$ \\
Induction chemotherapy & $66(97)$ \\
Initial diagnosis & \\
Adenocarcinoma & $33(49)$ \\
Squamous cell carcinoma & $16(24)$ \\
Undifferentiated large cell carcinoma & $2(3)$ \\
Undifferentiated non-small cell carcinoma & $17(25)$ \\
Positive nodes on mediastinoscopy (n) & \\
1 & $53(78)$ \\
2 & $14(21)$ \\
$3-4$ & $1(1)$ \\
\hline Data presented as n (\%), unless otherwise noted. &
\end{tabular}

\section{Feasibility Calculation}

VATS restaging met the prestudy criteria of feasibility in 27 of the 68 eligible cases $(40 \%)$. This group included 7 $(10 \%)$ restaging procedures that successfully sampled 3 negative mediastinal nodal stations, confirmed by absent

TABLE 2. Thoracic surgical complications $(n=66)$

\begin{tabular}{lccc}
\hline & \multicolumn{3}{c}{ Toxicity grade (n) } \\
\cline { 2 - 4 } Complication & $\mathbf{3}$ (Severe) & $\begin{array}{c}\mathbf{4} \text { (Life } \\
\text { threatening) }\end{array}$ & $\mathbf{5}$ \\
(Lethal)
\end{tabular}

residual nodal disease at thoracotomy (including the originally diseased nodal stations discovered at mediastinoscopy), 16 participants $(24 \%)$ who had 1 or more persistently positive mediastinal nodes sampled before thoracotomy, and 4 patients $(6 \%)$ with clinically occult pleural carcinomatosis (T4 disease).

Of the 68 subjects, $21(31 \%)$ did not have a successful VATS restaging procedure. The procedure was aborted in 11 owing to pleural space adhesions or tumor bulk $(\mathrm{n}=9)$, injury to the airway $(\mathrm{n}=1)$, or an inability to achieve adequate atelectasis $(\mathrm{n}=1)$. In 4 patients, 3 lymph node stations had biopsy cores taken thoracoscopically without evidence of tumor, but residual nodal disease was found at thoracotomy. Finally, fewer than 3 lymph node stations were sampled thoracoscopically with obliterated nodal stations and no nodal disease found by VATS restaging but N2 disease found at thoracotomy in 6 . These were considered VATS restaging failures. We could detect no statistical relationship between the primary tumor locations (upper vs lower lobe) or surgeon restaging experience and the likelihood of having a failed or inadequately sampled outcome.

In 26 patients $(38 \%)$, the prestudy success endpoint failed because fewer than 3 nodal stations had biopsy samples taken during restaging. Despite dissection of these mediastinal N2 stations by experienced surgeons during thoracotomy and lung resection, no nodal tissue was identified in at least $1 \mathrm{~N} 2$ station for each of these patients. The neoadjuvant chemotherapy and/or radiotherapy appeared to have obliterated all nodal tissue within the anatomic station, and only scar tissue remained. Of these 26 cases, 6 were mentioned in the previous paragraph as being a false-negative sampling of persistent nodal disease found at thoracotomy and were included in the VATS restaging failure group.

The remaining 20 subjects (29\% of the evaluable group) had a positive mediastinal node at mediastinoscopy, received neoadjuvant therapy, and had at least 1 nodal station with no remaining nodal tissue, but no nodal disease was found at thoracotomy with nodal dissection. Of these 20 patients, a VATS biopsy of the originally positive nodal stations was obtained in 14 patients $(70 \%)$, but a third nodal station had no nodal tissue. None of these 20 subjects had nodal disease found at the original site at thoracotomy, confirming downstaging of the disease. Because neoadjuvant therapy obliterated at least 1 required nodal station, the prestudy definition of success was flawed, because it was impossible to attain in some cases. Accordingly, it would be reasonable to reclassify these 20 cases as a part of a separate analysis.

Using these data, if the subgroup of participants with inadequate sampling were considered a VATS failure, the feasibility rate would be 27 of 68 or $40 \%$ (95\% CI, 28\%-52\%) and the false negative rate (VATS did not detect persistent nodal disease discovered at thoracotomy) would be $38.5 \%$ (95\% CI, 20\%-59.4\%). If the subgroup of patients with 
fewer than 3 nodal stations sampled but in which no persistent nodal disease was present at thoracotomy were considered VATS successes, the feasibility rate would be $(20+27) / 68$ or $69 \%(95 \%$ CI, $57 \%-80 \%)$, with a falsenegative rate of $33 \%(95 \% \mathrm{CI}, 17 \%-53 \%)$. Because the estimated feasibility rate of $69 \%$ is greater than the prespecified lower limit of continued interest (ie, $65 \%$ ), the feasibility of VATS restaging remains a question of interest for future investigation. However, because the lower limit of continued interest lies within the $95 \%$ CI of $57 \%$ to $80 \%$, our data failed to unequivocally establish the feasibility of VATS restaging.

\section{Effects of Radiotherapy and Chemotherapy}

Logistic regression analyses were conducted to examine the effect of radiotherapy (received vs not received) on VATS efficacy (success vs failure, and success vs empty nodal stations with negative thoracotomy). The analysis indicated that VATS failure using the prestudy definitions was associated with radiotherapy (Tables 3 and 4), although this was not dose related. Also, no statistically significant difference was found in the efficacy between the groups receiving 0 to 2 cycles of chemotherapy compared with those participants receiving greater than 2 cycles. In addition to the radiation effect, patients with adenocarcinoma had a significantly lower chance of VATS success than did patients with other histologic types.

\section{Anatomic Correlation With Restaging Failure}

An analysis of the location of persistently positive mediastinal nodes found at thoracotomy when the VATS restaging procedure failed to detect positive nodes is presented in Table 5. Nearly one half of all the false-negative nodes were in station $4 \mathrm{R}$.

\section{DISCUSSION}

The results of the present study have demonstrated that VATS could be a useful tool to detect or exclude persistent nodal disease, and VATS restaging might have a superior NPV in patients who do not receive radiotherapy as a part of their induction therapy. It proved particularly useful in

TABLE 3. VATS outcome stratified by receipt of radiotherapy or chemotherapy $(n=64 *)$

\begin{tabular}{lcccc}
\hline \multicolumn{1}{c}{ VATS outcome } & \multicolumn{4}{c}{ Patients (n) } \\
\hline Radiation dose (cGy) & None & $<5000$ & $5000-<6000$ & $\geq 60,000$ \\
Success & 11 & 5 & 5 & 2 \\
Failure & 5 & 6 & 6 & 4 \\
Inadequate sampling & 5 & 9 & 3 & 3 \\
Chemotherapy cycle & None & $1-2$ & $3-5$ & $6-8$ \\
Success & 1 & 8 & 10 & 4 \\
Failure & 0 & 8 & 7 & 6 \\
Inadequate sampling & 0 & 16 & 4 & 0 \\
\hline VATS, Videothoracoscopy. *Four patients with pleural carcinomatosis not included.
\end{tabular}

discovering occult pleural carcinomatosis $(6 \%$ in the present prospective trial) after neoadjuvant therapy for IIIA (N2) disease. Restaging thoracoscopy did have trouble reliably restaging the station $4 \mathrm{R}$ nodes.

Neoadjuvant therapy before surgical resection of locally advanced stage IIIA (N2) NSCLC improves survival for those patients who have had sterilization of their mediastinal nodes. ${ }^{2,3}$ More specifically, patients with persistent N2 disease at surgical resection have long-term survival similar to that of those treated nonoperatively. ${ }^{6}$ Mature analyses of both the Southwest Oncology Group 8805 study $^{2}$ and CALGB $8935 \mathrm{trial}^{3}$ showed that nodal downstaging is the most important predictor of long-term survival.

The mediastinal nodal downstaging rate of $21 \%$ to $50 \%$ seen in the 2 American Cooperative Group trials and the clinical significance of persistent disease emphasize the need to accurately restage the mediastinum after neoadjuvant therapy. Imaging alone has been unable to provide sufficient accuracy. Although PET scans have shown value in predicting persistent viable tumor in the primary tumor bed, they are unreliable in assessing the mediastinal nodes. The specificity for persistent mediastinal nodal disease in restaging PET scans has generally been $85 \%$ to $90 \%$, but the sensitivity has been disappointingly low at $50 \%$ to $60 \% .^{7-12}$ This might be because of the inability to detect a small focus of persistent disease within fibrosis or altered perfusion to the nodes that affect the PET tracer.

The inability to accurately predict persistent nodal disease by noninvasive imaging has led to the use of surgical restaging of the mediastinum. Although some investigators have reported an accuracy for repeat mediastinoscopy approaching that of initial mediastinoscopy, ${ }^{13,14}$ other large series have reported lower sensitivity and accuracy rates. A series of 104 repeat mediastinoscopies from 2 European academic centers in Spain and Belgium reported a sensitivity of $71 \%$, specificity of $100 \%$, and accuracy of $84 \%$. That series also reported a $2 \%$ rate of massive hemorrhage $(1$ aortic arch and 1 superior vena cava) and $1 \%$ mortality. ${ }^{5}$ A second combined German series of 104 repeat mediastinoscopies reported a sensitivity of $61 \%$ and accuracy of $88 \% .{ }^{15}$ A prospective trial comparing the accuracy of repeat mediastinoscopy and PETcomputed tomography fusion scans in 30 patients reported that the repeat surgical procedure was technically feasible but inaccurate owing to severe adhesions and fibrosis. ${ }^{7}$ The sensitivity to detect residual mediastinal disease in the present study was only $29 \%$, with an accuracy of $60 \%$. Fibrosis not only affects the accuracy of a repeat procedure, but also negatively affects the completeness of the mediastinal evaluation. ${ }^{4}$

It was unanticipated at the present trial's design that the use of high-dose neoadjuvant chemotherapy and radiotherapy would become widespread. The recognition that complete obliteration of all nodal tissue within a station of the 
TABLE 4. Statistics of logistic regression analysis of radiotherapy or chemotherapy on VATS success

\begin{tabular}{|c|c|c|c|c|}
\hline \multirow[b]{2}{*}{ Variable } & \multicolumn{2}{|c|}{ Success vs failure } & \multicolumn{2}{|c|}{ Success vs inadequate sampling } \\
\hline & $P$ value & OR $(95 \%$ CI $)$ & $P$ value & OR $(95 \%$ CI $)$ \\
\hline Radiotherapy (none vs yes) & .0525 & $4.9(1.0-24.6)$ & .0404 & $5.4(1.1-26.6)$ \\
\hline Age & .8226 & $1.0(0.9-1.1)$ & .4116 & $0.9(0.8-1.0)$ \\
\hline Gender (male vs female) & .1731 & $0.4(0.1-1.5)$ & .2178 & $0.4(0.1-1.7)$ \\
\hline Histologic type (adenocarcinoma vs other) & .0267 & $5.6(1.2-25.6)$ & .0349 & $4.9(1.1-21.1)$ \\
\hline Chemotherapy ( $\leq 2$ vs $>2$ cycles) & .7544 & $0.8(0.2-3.4)$ & .0059 & $0.1(0-0.5)$ \\
\hline Age & .7428 & $1.0(0.9-1.1)$ & 6509 & $1.0(0.9-1.1)$ \\
\hline Gender (male vs female) & .1068 & $0.3(0.1-1.3)$ & .3872 & $0.5(0.1-2.3)$ \\
\hline Histologic type (adenocarcinoma vs other) & .0598 & $3.8(0.9-15.6)$ & .0273 & $7.2(1.2-41.4)$ \\
\hline
\end{tabular}

"Success" defined as meeting protocol endpoints (ie, disease found in pleura, disease in mediastinal node, or 3 nodal stations sampled without disease); "failure" defined as not meeting protocol endpoints (ie, biopsy samples not obtained from 3 negative nodal stations or procedure aborted); "inadequate sampling" indicates inability to sample 3 nodal stations secondary to obliteration of nodal tissue. VATS, Videothoracoscopy; OR, odds ratio; $C I$, confidence interval.

lymph node map can occur after aggressive neoadjuvant therapy is 1 of the contributions of the present study. Twenty patients (nearly one third of the evaluable group) had histologically proven $\mathrm{N} 2$ disease at initial mediastinoscopy, with subsequent obliteration of all nodal tissue within that station at both VATS restaging and thoracotomy. Thus, we were compelled to perform a dual statistical analysis for the 26 participants $(39 \%)$ within this category. If we accepted the original protocol criteria that this subgroup would be defined as VATS failure for inadequate node sampling, the feasibility rate would only be $39.7 \%$. If we were willing to consider that this subgroup should be defined VATS success because no nodal disease was found at thoracotomy, the feasibility rate would be $69.1 \%$, which is marginally feasible. A subset analysis revealed that radiotherapy was linked to a lack of nodal tissue found within the fibrosis of these stations, although it was not dose related. Additional subset analysis also revealed a disproportionate rate of VATS failure in the station 4R position. Several causes for this outcome are possible, including the bulk of tumor being in the right upper lobe, preventing exposure of the $4 \mathrm{R}$ position; the relationship of the azygous-caval junction and the pulmonary artery to the nodal bed; the scar from the previous mediastinoscopy; and the radiation field effect on that location.

The strengths of the present prospective multi-institutional surgical study were considerable. All participants underwent pretreatment cervical mediastinoscopy with histologically

TABLE 5. Nodal stations when VATS negative and thoracotomy positive

\begin{tabular}{llr}
\hline Side & \multicolumn{1}{c}{ Station } & $\mathbf{n}$ \\
\hline Right & 2 Upper paratracheal & 2 \\
& 4 Lower paratracheal & 10 \\
& 7 Subcarinal & 3 \\
Left & 5 Aortopulmonary & 4 \\
& 6 Anterior mediastinal & 1 \\
& 9 Pulmonary ligament & 1 \\
\hline
\end{tabular}

VATS, Videothoracoscopy. confirmed N2 (not N3) disease by a CALGB thoracic surgeon. Neoadjuvant treatment was then set by each individual institution, with minimal criteria set by the protocol. Also, all participating surgeons underwent a quality assurance credentialing process.

The present trial also had weaknesses. The original trial design was created before our recognition that high-dose neoadjuvant therapy can completely obliterate all nodal tissue at certain stations. This placed us in a position of performing a post hoc analysis of the procedure because of the impossibility of sampling a node that no longer existed. Thus, the true feasibility likely lies somewhere between the 2 extremes of the data analysis we presented: the analysis by the original trial design (missing nodal tissue is failure) and the analysis by the assumption that missing nodal tissue could be considered equivalent to a sterilized nodal biopsy. The possibility of bias exists. If a surgeon failed to find nodal tissue during the thoracoscopic dissection of an N2 nodal station, it might have influenced the degree of surgical resection of the same nodal station at thoracotomy and lung resection. Considering the level of thoracic surgical expertise at the 10 participating centers, this seems unlikely. It is important to remember that 68 participants limited the power of the subanalysis. Also, our study was dominated by right-sided procedures $(76 \%)$. That limited observations of the technique on the left, but did allow us to further characterize the problem with sampling the station $4 \mathrm{R}$ position. Finally, the accrual of patients ended in 2003, and staging and restaging technology has improved since then. However, we believe the results from the present prospective trial still provide valuable data to thoracic surgeons who seek to individualize treatment of patients with stage IIIA (N2) NSCLC.

The technology has continued to advance since the period of the present prospective study. Many centers have now gained experience with minimally invasive endoscopic techniques such as transbronchial needle aspiration, endoesophageal ultrasound (EUS)-fine needle aspiration, and endobronchial ultrasound (EBUS)-fine needle aspiration. 
In 1 trial, the overall sensitivity and accuracy of 194 transbronchial needle aspiration samples without neoadjuvant therapy was $71 \%$ and $73 \%$, respectively. ${ }^{16}$ The accuracy was greatest in the station $4 \mathrm{R}$ position and least in the left paratracheal position. Although no large prospective studies have been published using EBUS specifically for restaging the mediastinum after neoadjuvant therapy for stage IIIA (N2) NSCLC, data are available for EUS. EUS-fine needle aspiration has been used to restage 19 patients treated with 3 cycles of platinum-based neoadjuvant chemotherapy without radiotherapy. ${ }^{17}$ The positive predictive value, NPV, sensitivity, specificity, and diagnostic accuracy was $100 \%$, $67 \%, 75 \%, 100 \%$, and $83 \%$, respectively. Another 128patient EBUS restaging series, however, showed a NPV of only $20 \% .{ }^{18}$ Another large series using EUS to stage untreated mediastinal nodal disease reported a sensitivity of only $72.6 \%,{ }^{19}$ and a meta-analysis of this same disease group reported a pooled sensitivity of $83 \%$, with a lower sensitivity of $39 \%$ to $75 \%$ for normal-size nodes. ${ }^{20}$ Needle samples from fibrotic nodes after high-dose chemoradiotherapy would likely have even lower sensitivity. Alternatively, a potential strategy could involve using a noninvasive technique such as EBUS/EUS at diagnosis and then saving mediastinoscopy for restaging after induction therapy. This might also allow the surgeon to assess the higher and contralateral nodes in a "virgin" mediastinum.

\section{CONCLUSIONS}

Thoracoscopic restaging of the mediastinum after neoadjuvant chemotherapy or radiotherapy is feasible in a prospective multi-institutional setting, although the feasibility decreased with radiotherapy or attempts to sample the $4 \mathrm{R}$ nodal station. This suggests that thoracoscopy could likely be combined with other new surgical staging techniques. EBUS and EUS could likely be used as an initial restaging modality guided by the results of a repeat PET-computed tomography scan. Patients found to have histologic confirmation of mediastinal nodes using these endoscopic procedures could then undergo additional nonoperative therapy. Those patients with negative mediastinal node biopsies would then have additional histologic confirmation of mediastinal nodal sterilization and the lack of pleural carcinomatosis by ipsilateral thoracoscopic restaging. Again, positive nodal disease might be treated nonoperatively. Thoracotomy and surgical resection might be offered to those patients with negative needle restaging followed by negative thoracoscopic restaging. Although intuitive, this restaging plan would need confirmation in an additional prospective randomized trial.

\section{References}

1. Sugarbaker DJ, Herndon J, Kohman LJ, Krasna MJ, Green MR, Cancer and Leukemia Group B Thoracic Surgery Group. Results of Cancer and Leukemia Group B protocol 8935: a multiinstitutional phase II trimodality trial for stage IIIA (N2) non-small cell lung cancer. J Thorac Cardiovasc Surg. 1995;109:473-83.
2. Albain KS, Rusch VW, Crowley JJ, Rice TW, Turrisi AT III, Weick JK, et al. Concurrent cisplatin/etoposide plus chest radiotherapy followed by surgery for stages IIIA (N2) and IIIB non-small-cell lung cancer: mature results of Southwest Oncology Group phase II study 8805. J Clin Oncol. 1995;13:1880-92.

3. Jaklitsch MT, Herndon JE III, DeCamp MM Jr, Richards WG, Kumar P, Krasna MJ, et al. Nodal downstaging predicts survival following induction chemotherapy for stage IIIA (N2) non-small cell lung cancer in CALGB protocol \#8935. J Surg Oncol. 2006;94:599-606.

4. Meersschaut D, Vernassen F, Brutel de la Riviere A, Knaepen PJ, Van den Bosch JM, Vandershueren R. Repeat mediastinoscopy in the assessment of new and recurrent lung neoplasm. Ann Thorac Surg. 1992;53:120-2.

5. De Waele M, Serra-Mitjans M, Hendriks J, Lauwers P, Belda-Sanchis J, Van Schil P, et al. Accuracy and survival of repeat mediastinoscopy after induction therapy for non-small cell lung cancer in a combined series of 104 patients. Eur J Cardiothorac Surg. 2008;33:824-8.

6. Bueno R, Richards WG, Swanson SJ, Jaklitsch MT, Lukanich JM, Mentzer SJ, et al. Nodal stage after induction therapy for stage IIIA lung cancer determines patient survival. Ann Thorac Surg. 2000;70:1826-31.

7. De Leyn P, Stoobants S, Dewever W, Lerut T, Coosemans W, Decker G, et al. Prospective comparative study of integrated PET-CT with remediastinoscopy in the assessment of residual mediastinal disease after induction chemotherapy for mediastinoscopy proven stage IIIA-N2 non-small cell lung cancer. J Clin Oncol. 2006;24:3333-9.

8. Akhurst T, Downey RJ, Ginsberg MS, Gonen M, Bains M, Korst R, et al. An initial experience with FDG-PET in the images of residual disease after induction therapy for lung cancer. Ann Thorac Surg. 2002;73:259-66.

9. Ryu JS, Choi NC, Fischman AJ, Lynch TJ, Mathisen DJ. FDG-PET in staging and restaging non-small cell lung cancer after neoadjuvant chemoradiotherapy: correlation with histopathology. Lung Cancer. 2002;35:179-87.

10. Cerfolio RJ, Ojha B, Mukherjee S, Pask AH, Bass CS, Katholi CR. Positron emission tomography scanning with 2-fluoro-2-deoxy-D-glucose as a predictor of response of neoadjuvant treatment for non-small cell carcinoma. $J$ Thorac Cardiovasc Surg. 2003;125:938-44.

11. Port JL, Kent MS, Korst RJ, Keresztes R, Levin MA, Altorki NK. Positron emission tomography poorly predicts response to preoperative chemotherapy in nonsmall cell lung cancer. Ann Thorac Surg. 2004;77:254-9.

12. Hellwig D, Graeter TP, Ukena D, Georg T, Kirsch CM, Schafers HJ. Value of F-18-fluorodesoxyglucose positron emission tomography after induction therapy of locally advanced bronchogenic carcinoma. J Thorac Cardiovasc Surg. 2004; 128:802-9.

13. Pauwels M, Van Schil P, De Backer W, Van den Brande F, Eyskens E. Repeat mediastinoscopy in the staging of lung cancer. Eur J Cardiothorac Surg. 1998;14:271-3.

14. Granone P, Van Schil P. Letter to editor: restaging patients with N2 (stage IIIa) non-small cell lung cancer after neoadjuvant chemoradiotherapy: a closer look at redo mediastinoscopy. J Thorac Cardiovasc Surg. 2007;133:275-6.

15. Marra A, Hillejan L, Fechner S, Stamatis G. Remediastinoscopy in restaging of lung cancer after induction therapy. J Thorac Cardiovasc Surg. 2008;135:843-9.

16. Patelli M, Lazzari A, Poletti V, Trisolini R, Cancellieri A, Lacava N, et al. Role of fiberscopic transbronchial needle aspiration in the staging of $\mathrm{N} 2$ disease due to non-small cell lung cancer. Ann Thorac Surg. 2002;73:407-11.

17. Annema J, Veselic M, Versteegh MIM, Willems LNA, Rabe KF. Mediastinal restaging: EUS-FNA offers a new perspective. Lung Cancer. 2003;42:311-8.

18. Herth FJF, Annema JT, Eberhardt R, Yasufuku K, Ernst A, Krasnik M, et al. Endobronchial ultrasound with transbronchial needle aspiration for restaging the mediastinum in lung cancer. J Clin Oncol. 2008;26:3346-50.

19. Witte B, Neumeister W, Huertgen M. Does endoesophageal ultrasound-guided fine-needle aspiration replace mediastinoscopy in mediastinal staging of thoracic malignancies? Eur J Cardiothorac Surg. 2008;33:1124-8.

20. Micames CG, McCrory DC, Pavey DA, Jowell PS, Gress FG. Endoscopic ultrasound-guided fine needle aspiration for non-small cell lung cancer staging: a systematic review and meta-analysis. Chest. 2007;131:539-48.

\section{APPENDIX}

The following institutions participated in the present study: Cedars-Sinai Medical Center, Los Angeles, Calif, Alan T. Lefor; Dana-Farber Cancer Institute, Boston, Mass, Harold J. Burstein, MD, PhD, supported by grant CA32291; Duke University Medical Center, Durham, NC, Jeffrey Crawford, MD, supported by grant CA47577; 
Massachusetts General Hospital, Boston, Mass, Jeffrey W. Clark, MD, supported by grant CA32291; Southeast Cancer Control Consortium, Inc CCOP, Goldsboro, NC, James N. Atkins, MD, supported by grant CA45808; State University of New York Upstate Medical University, Syracuse, NY, Stephen L. Graziano, MD, supported by grant CA21060; University of Iowa, Iowa City, Iowa, Daniel A. Vaena,
MD, supported by grant CA47642; University of Maryland Greenebaum Cancer Center, Baltimore, Md, Martin Edelman, MD, supported by grant CA31983; University of Minnesota, Minneapolis, Minn, Bruce A. Peterson, MD, supported by grant CA16450; University of Missouri/Ellis Fischel Cancer Center, Columbia, Mo, Michael C. Perry, MD, supported by grant CA12046. 